\title{
Ultrasound Assessment of the Internal Anal Sphincter in Women with Fecal Incontinence and Posterior Vaginal Wall Prolapse (Rectocele)
}

\author{
${ }^{1}$ Abdel Karim M EI Hemaly, ${ }^{1}$ lbrahim M Kandil, ${ }^{2}$ Asim Kurjak \\ ${ }^{3}$ Laila AS Mousa, ${ }^{1}$ Hossam H Kamel, ${ }^{4}$ Ahmad G Serour \\ ${ }^{1}$ Professor, Department of Obstetrics and Gynecology, Faculty of Medicine, Al Azhar University, Cairo, Egypt \\ ${ }^{2}$ Professor, Department of Obstetrics and Gynecology, Medical School, University of Zagreb, Croatia \\ ${ }^{3}$ P rofessor, Department of Pathology, Faculty of Medicine, Al Azhar University, Cairo, Egypt \\ ${ }^{4}$ Lecturer, Department of Obstetrics and Gynecology, Faculty of Medicine and International Islamic and Population Center, Al Azhar \\ University, Cairo, Egypt
}

Correspondence: Abdel Karim M El Hemaly, Professor, Department of Obstetrics and Gynecology, Faculty of Medicine Al Azhar University, 3 Emad El Din Kamel St Nasr City-11371, Cairo, E gypt, Phone: +202 22607085, +202 24011780, +202 010 1577969, Fax: +202 25893755, e-mail: profakhemaly@ hotmail.com

\begin{abstract}
Rupture of the internal anal sphincter (IAS) causes its weakness and it will not withstand increases of abdominal pressure, and fecal incontinence (FI) will occur. Recently, we put forward a novel concept on the physiology of defecation. Defecation is divided into two stages: First stage before training and second stage starts at the age of about 2 years, when the mother starts to teach her child how to hold up himself. This is gained by maintaining high alpha-sympathetic tone at the IAS, thus keeping it closed all the time till there is a need to pass stool or flatus, and the time and place are convenient. On defecation, six neuromuscular actions take place under the control of the CNS: (1) The person will relax the external anal sphincter, (2) he will lower the gained high alpha-sympathetic tone at the IAS, thus opening the anal canal, (3) he will relax the pelvic floor muscles bringing the rectum and the anal canal into one axis, (4) the abdominal and diaphragmatic muscles contract to increase the abdominal pressure, (5) the muscles of the distal colon and rectum contract pushing the stool, (6) sequential contractions of the three parts of the external anal sphincter (EAS) that squeeze any residual contents in the anal canal. Thus, the anal canal is closed and empty under normal circumstances. The IAS is a collageno-muscular tissue cylinder that surrounds the anal canal.

The IAS is intimately related to the posterior vaginal wall, and the vagina is over stretched in labor, childbirth trauma affects both the posterior vaginal wall and the IAS. Rupture of the collagen sheet of the IAS which causes its weakness is better demonstrated by imaging by 3D US.

Normal vagina is a cylinder of collageno-elastic-muscular tissues. Its strong collagen sheet is responsible for keeping it in its normal upward position. Labors cause redundancy and weakness of the vaginal walls with subsequent prolapse; and lacerations of the IAS which is closely related to the posterior vaginal wall leading to $\mathrm{FI}$.
\end{abstract}

Keywords: Three-dimensional ultrasound (3D US), Fecal incontinence (FI), Internal anal sphincter (IAS), External anal sphincter (EAS), Collagen, Rectocele, Central nervous system (CNS).

\section{INTRODUCTION}

The role of the internal anal sphincter (IAS) in keeping fecal continence and in the mechanism of defecation has not been fully understood. A Iso, the structure of the IAS has not been thoroughly studied and investigated. ${ }^{1-7}$

Recently, we put forward a novel concept on the physiology of defecation and the structure of the IAS. ${ }^{1}$ D efecation is divided into two stages. First stage, in infancy before training, once the rectum is distended the rectal muscles contract expelling its contents, and the external anal sphincter relaxes allowing passage of stool. The second stage, the mother starts to teach her child at about the age of 2 years, how to hold up till appropriate time and place are available. This is gained by maintaining high al pha-sympathetic tone at the IA S that keeps it closed all the time. When there is a desire to pass stool and the time and place are convenient, under the control of the central nervous system (CNS) six neuromuscular actions take place:

1. The person will lower the high alpha-sympathetic tone at the IAS relaxing the sphincter and opening the closed empty anal canal

2. He will relax the external anal sphincter which is a striated muscle innervated by the pudendal nerve

3. He will relax the levator ani muscles, to bring the rectum and anal canal in one axis instead of the acute angle between them

4. The abdominal and diaphragmatic muscles contract increasing the abdominal pressure helping in pushing the stool through the rectum into the opened anal canal to outside 
5. The muscles of the distal colon and rectum contract propelling the stool

6. Sequential contractions of the three parts of the external anal sphincter, the deep, the superficial and the subcutaneous parts to squeeze any residual contents from the anal canal.

Under normal circumstances, the anal canal is kept empty and closed. The IAS is a collageno-muscular tissue cylinder that surrounds the anal canal and is innervated by alphasympathetic nerves from the hypogastric nerves. It is surrounded in its lower part by the external anal sphincter which is a striated muscle innervated by the pudendal nerve (Figs 1 to 3 ).

The IAS is in close relation to the posterior vaginal wall which stretches very much during labor. Prolonged labor, difficult, multiple frequent labors cause overstretching of the posterior vaginal wall, leading to flabbiness of the vagina with subsequent falling down of the redundant vaginal wall prolapse (rectocele). The redundancy of the vaginal wall is the result of rupture of its collagenous sheet. The rupture may affect the intimately related IAS with subsequent $\mathrm{FI}$. The rupture in the IAS affects the collagenous sheet as seen in Figures 9 and 10. This is a surgical biopsy taken during 'U rethro-A noVaginoplasty' operation and stained with Masson trichrome

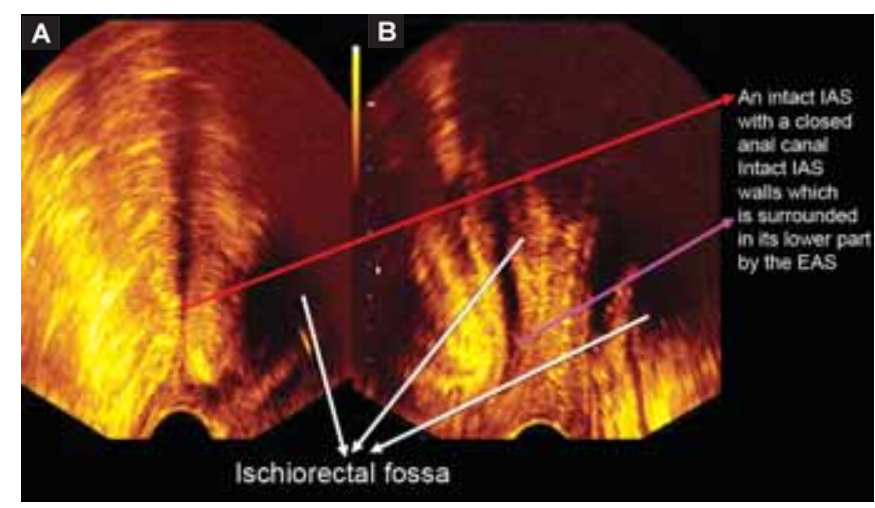

Fig. 1: Transvaginal 3D US picture: (A) Coronal section showing a normal IAS with intact sphincter walls and closed lumen. The IAS is surrounded in its lower part by the EAS; $(B)$ the presence of an angle between the rectum above and the closed anal canal below

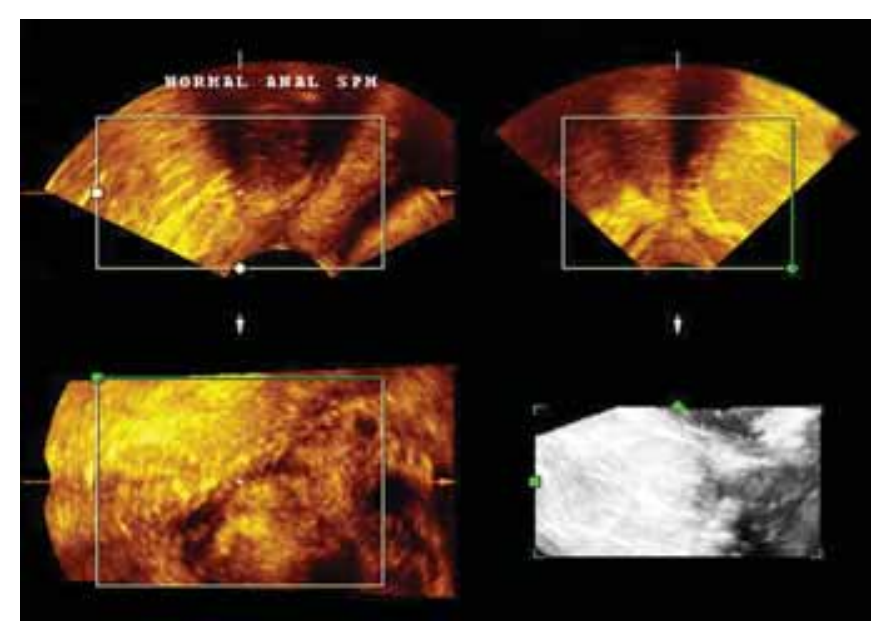

Fig. 2: Transvaginal 3D US pictures, coronal and cross section showing a normal IAS with a closed lumen and intact walls. It is surrounded in its lower part by the EAS

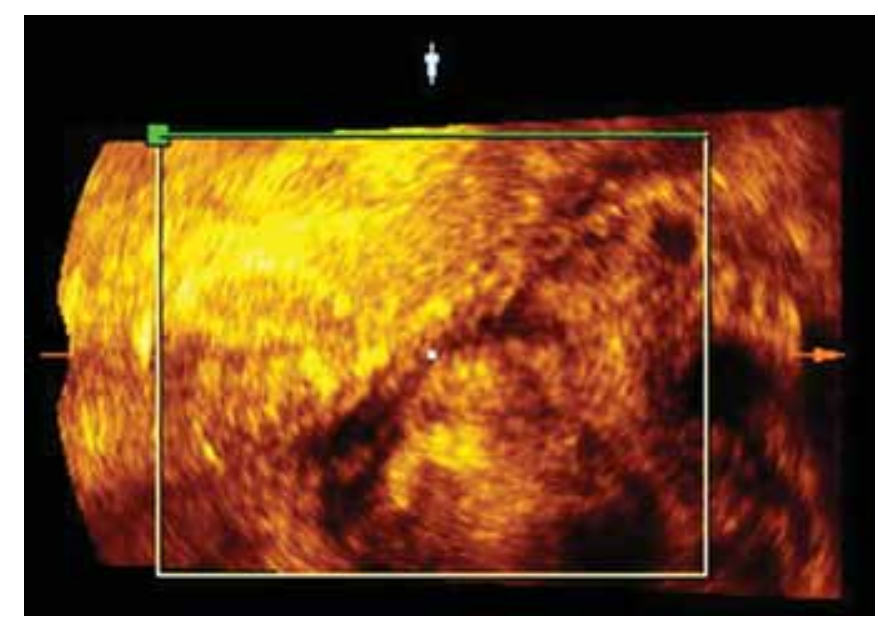

Fig. 3: Cross section of 3D US picture of normal IAS showing the anal canal closed and surrounded by the IAS which is surrounded in its lower part by the EAS

acetate stain which stains collagen fibers greenish-blue and muscle fibers red. The figures show torn collagen sheet in women suffering from $\mathrm{FI}$ because of torn IAS. ${ }^{1-3}$

\section{AIM}

Imaging by three-dimensional ultrasound (3D US) which gives volumetric serial planes of images.

\section{METHOD}

Using a vaginal probe multifrequent 5 to $7.5 \mathrm{M} \mathrm{Hz}$ (General Electric), integrated 3D-4D Unit (GE V olosone), 730 Pro V machine is done for 40 patients with $\mathrm{Fl}$ and 10 continent women.

\section{RESULTS}

The anal canal is closed in normal women, with intact IAS as seen in Figures 1 to 3. In women suffering from $\mathrm{FI}$ the anal canal is wide and open with torn IA S wall as seen in Figures 4 to 8.

\section{DISCUSSION}

The internal anal sphincter (IAS) is a collageno-muscular tissue cylinder that surrounds the anal canal innervated by alphasympathetic nerve supply from the hypogastric nerves (see Figs 1 to 3). It is surrounded in its lower part by the EAS which is a striated muscle innervated by the pudendal nerve. Damage of the IA S causes dilation of the anal canal. 0 pen and dilated anal canal allows the rectal contents to enter the anal canal with subsequent fecal incontinence. So, we can more correctly say that the first cause of $\mathrm{FI}$ is anal sphincter damage, traumatic injury to one and/or both anal sphincters, IA S, EAS (Fig. 11). A lso we can add, anal intercourse as a traumatic cause of anal sphincter injury.

Vaginal prolapse, anterior wall and posterior wall, is quite common, especially after frequent multiple labors, difficult and instrumental labors. Childbirth trauma causes redundancy, 


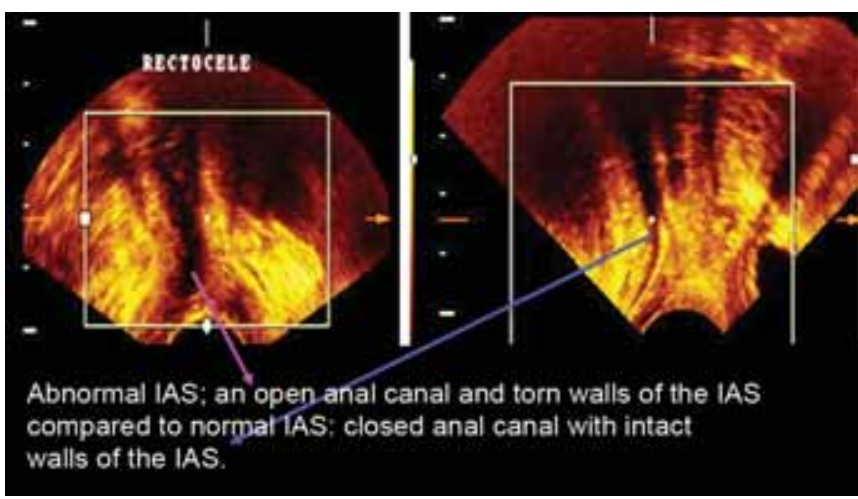

Fig. 4: Comparison between torn IAS with an opened anal canal on the left as compared with an intact IAS with a closed anal canal on the right as seen by 3D US images

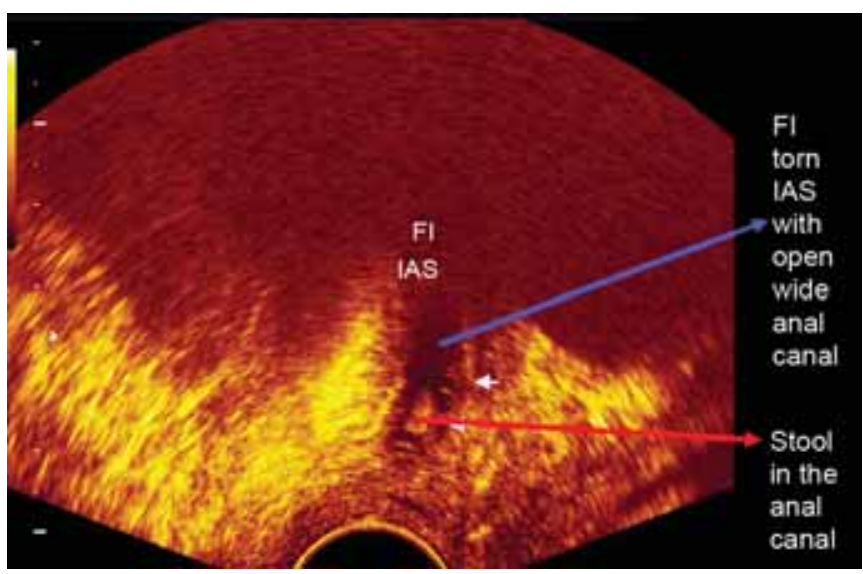

Fig. 5: Imaging by 3D US transvaginally of a patient with FI, the figure shows torn IAS with irregular cut walls and an open anal canal with a piece of stool in the anal canal
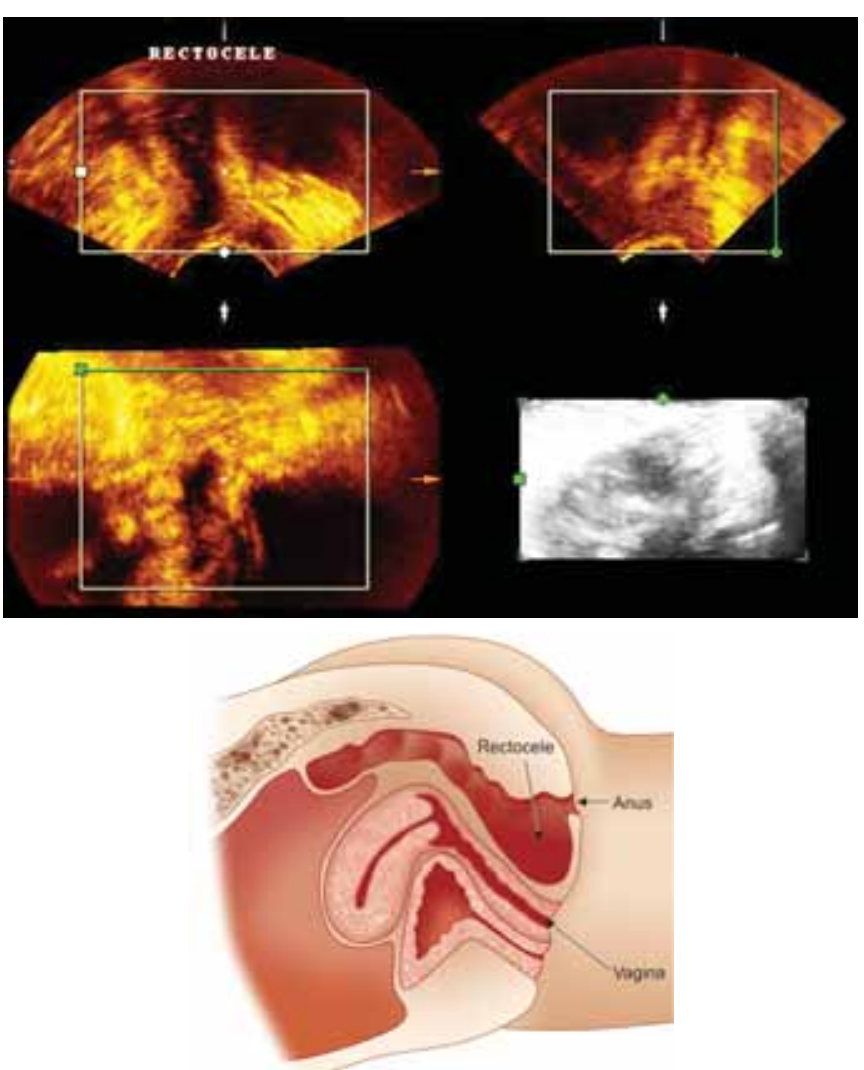

Fig. 6: Imaging by 3D US transvaginally, a case of rectocele that shows damaged IAS with torn walls and open anal canal

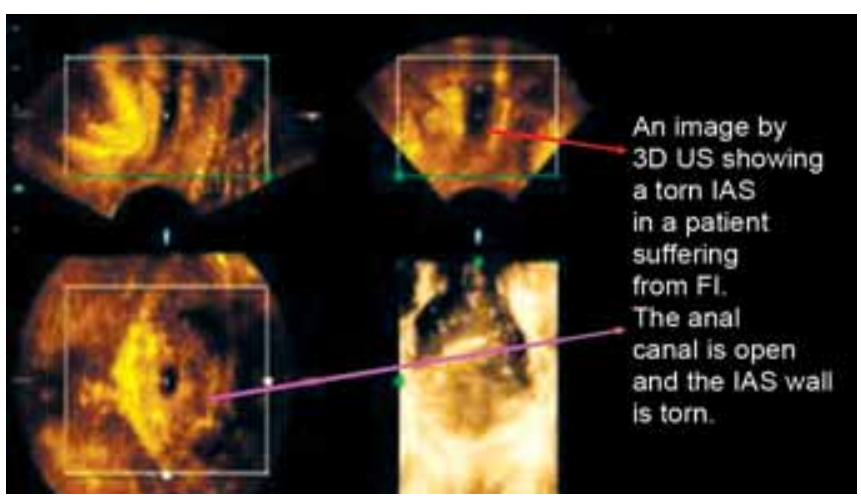

Fig. 7: An image by 3D US showing a torn IAS in a patient suffering from FI. The anal canal is open and the IAS wall is torn

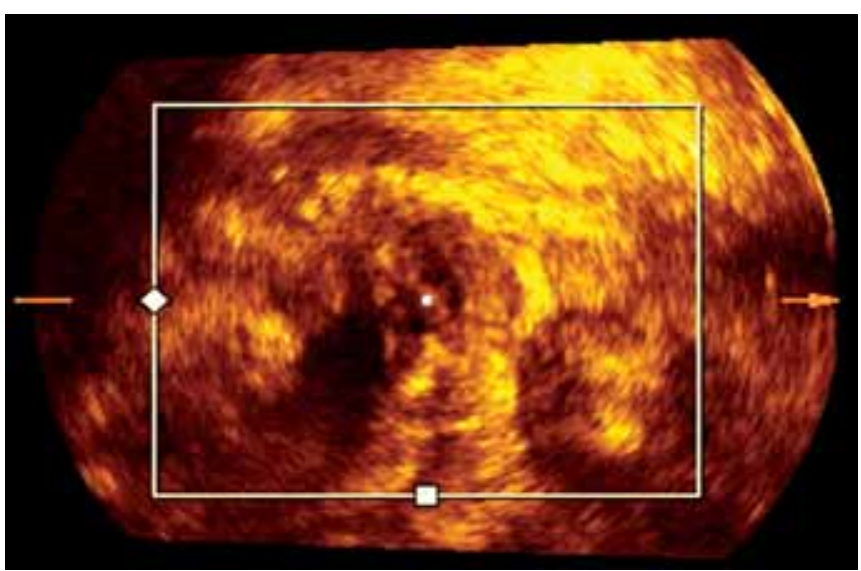

Fig. 8: Torn IAS seen, the damage is mainly in the collagenous sheet with muscle fibers intact. Note that the anal canal is opened. A patient who was suffering from FI, imaged by 3D US transvaginally, showing in the cross section torn IAS. The damage is mainly in the collagenous sheet leaving the muscle fibers intact. Kindly notice an open anal canal

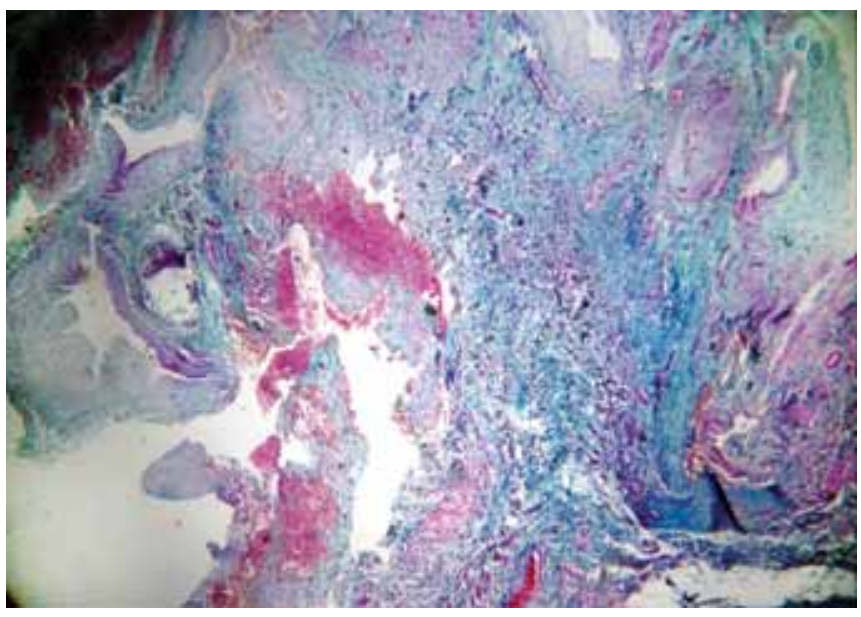

Fig. 9: Histopathology, specimen taken during surgery for $\mathrm{FI}$ in a patient with torn IAS stained with Masson trichrome acetate which stains collagen greenish-blue and muscle red. It shows torn collagenous sheet of the IAS

flabbiness and rupture of the collagenous sheet of the vagina leading to its prolapse. At the same time, it causes rupture of the internal urethral sphincter (IUS) leading to stress urinary incontinence (SUI); and rupture of the IAS leading to fecal incontinence. 


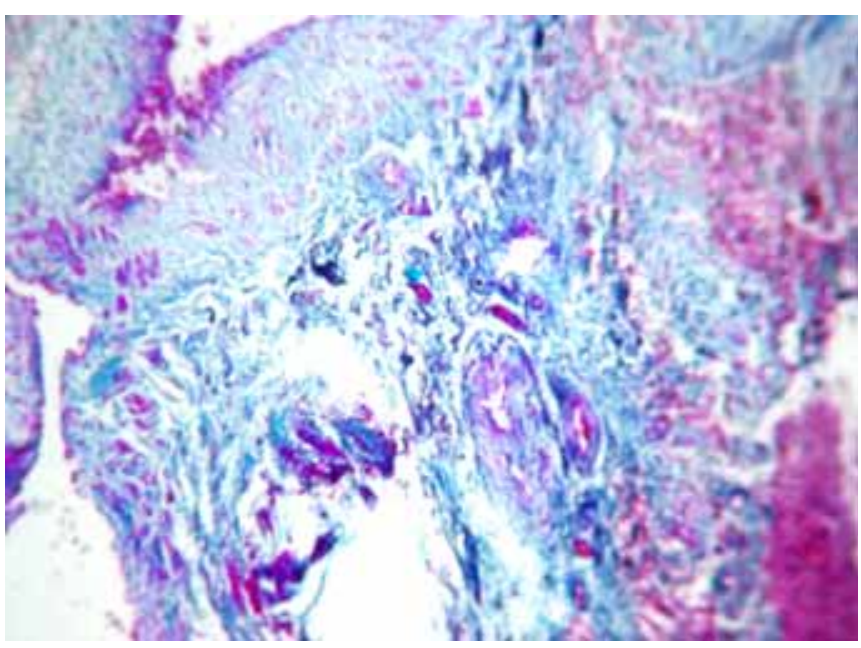

Fig. 10: Surgical specimen from the IAS of a patient with FI stained with Masson's trichrome acetate showing torn collagenous layer of the IAS

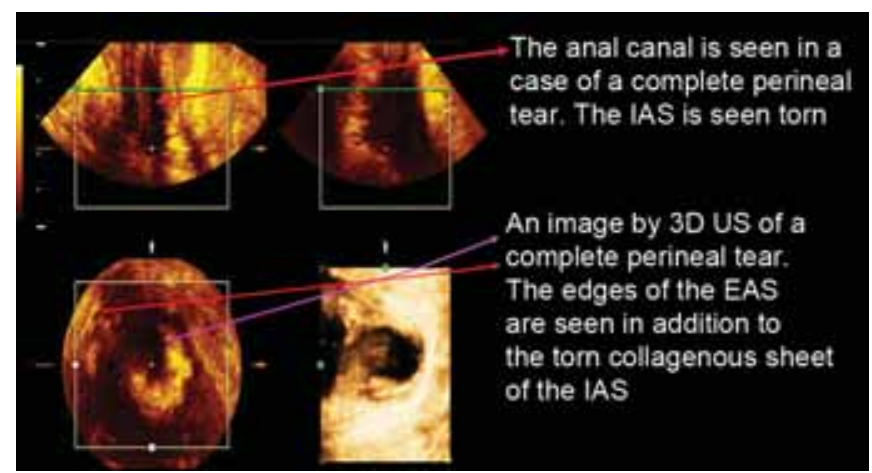

Fig. 11: Imaging by 3D US transvaginally of a patient, who is suffering from complete perineal tear, showing the anal canal is open and the IAS torn in addition to the torn edges of the EAS

\section{CONCLUSION}

Pelvic collagen is an essential support of the pelvic organs. It is an essential part of the constituents of the IUS, IAS, pelvic ligaments and the vagina. Rupture and weakness of the pelvic collagen is the cause of SUI, FI and genital prolapse. If the rupture affects the collagen of the IUS it will cause SUI. If the rupture affects the collagen of the IAS it will lead to $\mathrm{FI}$. If the rupture affects the collagen of the vagina it will lead to vaginal prolapse. If the damage affects the pelvic ligaments (uterosacral and transverse cervical ligaments) it will lead to uterine descent and/or vault prolapse.

\section{REFERENCES}

1. Hemaly A bdel Karim M EI, M ousa Laila A, K andil Ibrahim M, Sokkary Fatma S El, Serour A hmad G, Hussein Hossam. Fecal incontinence: A novel concept. The role of the internal anal sphincter (IAS) in defecation and fecal incontinence. Gynaecologia et Perinatologia A pril-J une 2010;19(2):73-128.

2. Hemaly $A$ bdel Karim M El, K andil Ibrahim M, Kurjak A sim, Serour A hmad G, M ousa Laila A, Zaied A mr M, El Sheikha $K$ halid Z. I maging the internal urethral sphincter and the vagina in normal women and women suffering from stress urinary incontinence and vaginal prolapse. Gynaecologia et Perinatologia, October-D ecember 2009;18(4):169-286.

3. Hemaly A bdel Karim M EI, M ousa Laila A, Kandil Ibrahim M, Sokkary FatmaS El, Serour A hmad G, H ussein Hossam. Surgical treatment of stress urinary incontinence (SUI), fecal incontinence (FI) and vaginal prolapse by a novel operation, "urethroano-vaginoplasy" stress urinary incontinence (SUI), fecal incontinence (FI) and vaginal prolapse: A novel concept on their pathophysiology and their surgical treatment. Gynaecologia et Perinatologia July-September 2010;19(3):129.

4. Diane France-B orello, Burgio K athryn L, Richter HE Holly E, et al. $U$ rinary incontinence in primiparous women. For the Pelvic Floor Disorders, Network, Fecal and Obstet \& Gynec 2006;108(4):863-72.

5. Pinta TM, K ylanpaa ML, Salmi TK, et al. Primary sphincter repair: A re the results of the operation good enough? Dis Colon Rectum 2004;47:18-23.

6. Halverson A L, Hull TL . L ong-term outcome of overlapping anal sphincter repair. Dis Colon Rectum 2002;45:345-48.

7. Sultan AH, Thakar R. Lower genital tract and anal sphincter trauma. B est Pract Res Clin Obstet Gyecol 2002;16:99-115. 